\title{
ON ALMOST-ANALYTIC FUNCTIONS, TENSORS AND INVARIANT SUBSPACES
}

\author{
SHUN-ICHI TACHIBANA AND SATOSHI Kotō
}

(Received February 15, 1962)

0. Introduction. In previous papers [2], [6], ${ }^{1)}$ we introduced the notion of $\Phi$-tensors as a generalization of analytic tensors. The main purpose of this paper is to introduce the notion of almost-analytic function and almost-analytic submanifold and to define an operator acting on tensor fields defined on an invariant subspace.

In $\S 1$ and $\S 2$ we define almost-analytic functions and almost-analytic submanifolds in an almost-complex space. A new $\Phi$-operator on an invariant subspace of the manifold admitting a tensor fields of type $(1,1)$ is defined in $\S 4$, after preliminary facts are given in $\S 3$. Some formulas about the $\Phi$-operator are given in $\$ 5$ and we devote $\$ 6$ to Kählerian spaces. The results of all sections except $\S 6$ are independent from connection or metric.

1. Almost-analytic functions. Consider an $n$-dimensional differentiable manifold admitting a tensor field whose components with respect to local coordinates $\left\{x^{\lambda}\right\}$ are $\varphi_{\mu}^{\lambda{ }^{2)}}$. A tensor field $T_{(\mu)}^{(\lambda)} \equiv T_{\mu_{q} \ldots \mu_{1}}{ }^{\lambda_{p} \ldots \lambda_{1}}$ is called to be pure in $\lambda_{i}, \mu_{j}$ or briefly to be $\mathfrak{p}\left(\lambda_{i}, \mu_{j}\right)$ if it commutes with $\varphi_{\mu}^{\lambda}$ in $\lambda_{i}$, $\mu_{j}$ i. e. it holds that

$$
{\varphi_{\mu}}_{\mu_{j}}{ } T_{\mu q \ldots \sigma \ldots \mu_{1}}{ }^{(\lambda)}={\varphi_{\sigma}}^{\lambda_{t}} T_{(\mu)}{ }^{\lambda_{p} \ldots \sigma \ldots \lambda_{1}} .
$$

A nalogously $T_{(\mu)}^{\left({ }^{(1)}\right.}$ is called to be $\mathfrak{p}\left(\lambda_{i}, \lambda_{j}\right)$ or $\mathfrak{p}\left(\mu_{i}, \mu_{j}\right)$ if

$$
\varphi_{\sigma}{ }^{\lambda_{i}} T_{(\mu)}{ }^{\lambda_{p} \ldots \sigma \ldots \lambda_{j} \ldots \lambda_{1}}={\varphi_{\sigma}}^{\lambda_{j}} T_{(\mu)}^{\lambda_{p} \ldots \lambda_{i} \ldots \sigma \ldots \lambda_{1}},
$$

or

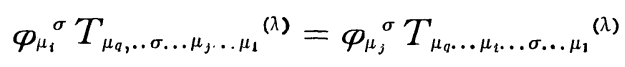

is valid respectively. If a tensor is pure in every pair of its indices, then it is called a pure tensor. We consider for convenience sake the covariant vector and the contravariant vector as pure tensors.

A tensor field $T_{(\mu)}^{(\lambda)}$ is called to be hybrid in $\lambda_{i}, \mu_{j}$ or briefly to be $\mathfrak{h}\left(\lambda_{i}, \mu_{j}\right)$ if it anticommutes with $\varphi_{\mu}{ }^{\lambda}$ in $\lambda_{i}$, $\mu_{j}$ i.e. it holds that

$$
{\varphi_{\mu}}^{\sigma} T_{\mu_{q} \ldots \sigma \ldots \mu_{1}}^{(\lambda)}=-\varphi_{s}{ }^{\lambda_{i}} T_{(\mu)}{ }^{\lambda_{p} \ldots \sigma \ldots \lambda_{1}} .
$$

1) The number in brackets refers to the the Bibliography at the end of the paper.

2) $\lambda, \mu, \nu, \cdots=1,2, \cdots, n$. As to the notations we follow Tachibana, S., [6]. 
Analogously $\mathfrak{h}\left(\lambda_{i}, \lambda_{j}\right)$ and $\mathfrak{h}\left(\mu_{i}, \mu_{j}\right)$ are defined.

The $\Phi$-operator is defined for pure tensors as follows,

$$
\begin{aligned}
\Phi_{\nu} T_{(\mu)}^{(\lambda)}= & \varphi_{\nu}^{\sigma} \partial_{\sigma} T_{(\mu)}^{(\lambda)}-\partial_{\nu}^{*} T_{(i)}^{(\lambda)} \\
& +\sum_{i=1}^{q}\left(\partial_{\mu_{i}} \varphi_{\nu}^{\sigma}\right) T_{\mu_{q} \ldots \sigma \ldots \mu_{1}}{ }^{(\lambda)} \\
& +\sum_{j=1}^{p}\left(\partial_{\nu}{\varphi_{\sigma}}^{\lambda_{j}}-\partial_{\sigma}{\varphi_{\nu}}_{\nu}^{\lambda_{j}}\right) T_{(\mu)}{ }^{\lambda_{p} \ldots \sigma \ldots \lambda_{1}},
\end{aligned}
$$

where $\partial_{\sigma}=\partial / \partial x^{s}$ and

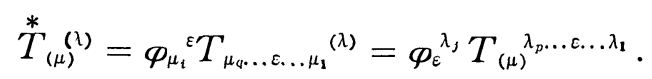

This operator yields from a pure tensor of type $(p, q)$ a tensor of type $(p, q+1)$. The definition is independent from connection or metric, so it is defined on differentiable manifolds admitting a tensor field of type $(1,1)$.

The following equations are known ${ }^{3}$,

$$
\begin{aligned}
& \Phi_{\nu} v^{\lambda}=-\underset{v}{\mathfrak{f}} \varphi_{\nu}^{\lambda}, \quad \Phi_{\nu} \delta_{\mu}^{\lambda}=0, \\
& \Phi_{\nu} \varphi_{\mu}^{\lambda}=N_{\nu \mu}^{\lambda},
\end{aligned}
$$

where $\underset{v}{\mathfrak{f}}$ denotes the operator of Lie derivation with respect to $v^{\lambda}$ and $N_{\nu \mu}^{\lambda}$ is the so-called Nijenhuis tensor defined by

$$
N_{\nu \mu}{ }^{\lambda}=\varphi_{\nu}{ }^{\sigma} \partial_{\sigma} \varphi_{\mu}{ }^{\lambda}-\varphi_{\mu}{ }^{\sigma} \partial_{\sigma}{\varphi_{\nu}}^{\lambda}+\varphi_{\sigma}^{\lambda}\left(\partial_{\mu} \varphi_{\nu}{ }^{\sigma}-\partial_{\nu}{\varphi_{\mu}}^{\sigma}\right)^{4)}
$$

Now we consider an almost-complex space which is a differentiable munifold admitting a tensor field $\varphi_{\mu}^{\lambda}$ such that $\varphi_{\sigma}{ }^{\lambda} \varphi_{\mu}{ }^{\sigma}=-\delta_{\lambda}{ }^{\mu}$. In the space a pure tensor $T_{(\mu)}^{(\lambda)}$ satisfying $\Phi, T_{(}()^{(\lambda)}=0$ is called to be almost-analytic. If there exists a local function $g$ such that $\varphi_{\lambda}{ }^{\varepsilon} \partial_{\varepsilon} f=\partial_{\lambda} g$ for a local function $f$, then we shall call $f$ an almost-analytic function of $x^{\lambda}$ or a locally almost-analytic function and call $g$ its associated function. If such a function $f$ is defined globally, then we call it an almost-analytic function. In a complex manifold i. e. an almostcomplex space with vanishing Nijenhuis tensor, we say "analytic" instead of "almost-analytic".

For a local function $f$ we have

$$
\begin{aligned}
\Phi_{\nu} \partial_{\lambda} f & =\varphi_{\nu}{ }^{\varepsilon} \partial_{\varepsilon} \partial_{\lambda} f-\partial_{\nu}\left(\varphi_{\lambda}{ }^{\varepsilon} \partial_{\varepsilon} f\right)+\left(\partial_{\lambda} \varphi_{\nu}{ }^{\varepsilon}\right) \partial_{\varepsilon} f \\
& =\partial_{\lambda}\left(\varphi_{\nu}{ }^{\varepsilon} \partial_{\varepsilon} f\right)-\partial_{\nu}\left(\varphi_{\lambda}{ }^{\varepsilon} \partial_{\varepsilon} f\right)
\end{aligned}
$$

so $\Phi \partial_{\lambda} f=0$ is equivalent to the existence of a local function $g$ such that $\partial_{\nu} g$ $=\varphi_{v}{ }^{\varepsilon} \partial_{\varepsilon} f$.

3) Tachibana, S., [6].

4) Nijenhuis, A., [4]. 
Hence a locally almost-analytic function $f$ is characterized by $\Phi_{\nu} \partial_{\lambda} f=0$. For any $v^{\lambda}$ and $u_{\lambda}$ we have the equation

$$
\left.v^{\sigma} \Phi_{\nu} u_{\sigma}+u_{\sigma} \Phi_{\iota} v^{\sigma}=\varphi_{v}^{\varepsilon} \partial_{\varepsilon}\left(v^{\sigma} u_{\sigma}\right)-\partial_{\nu}{ }^{*} v^{\sigma} u_{\sigma}\right)
$$

Hence if $v^{\lambda}$ is almost-analytic, then $v^{\sigma} \partial_{\sigma} f$ is almost-analytic for any almostanalytic $f$ and if $u_{\lambda}$ is almost-analytic, then $u_{\sigma} v^{\sigma}$ is almost-analytic for any almost-analytic $v^{\lambda}$.

We have known much about almost-analytic tensors in almost-Hermitian spaces. In $\$ 1$ and $\S 2$ we give some theorems about them which are independent from connection or metric.

First we have known the following

LEMMA 1. (Yano, K.) Let $f$ be a scalar function on a compact almostcomplex space. If the form $\eta=\eta_{\nu} d x^{v}$ defined by

$$
\eta_{v}=\phi^{\varepsilon} \partial_{\varepsilon} f
$$

is closed, then it is constant.

Proof. As such a space always admits a positive definite Riemannian metric tensor $g_{\mu \lambda}$ which is $\mathfrak{h}(\lambda, \mu)$, we denote by $\nabla_{\nu}$ the operator of the Riemannian covariant derivation and make use of it. From (1.1) we have $\nabla \lambda f=$ $-\varphi_{\lambda}{ }^{\varepsilon} \eta_{i}$. Applying $g^{i \lambda} \nabla_{\mu}$ to this we have

$$
g^{\mu \lambda} \nabla_{\mu} \nabla_{\lambda} f=-g^{\mu \lambda}\left(\nabla_{\mu} \varphi_{\lambda}\right) \eta_{\dot{\varepsilon}} .
$$

Substituting (1. 1) into the right hand side we get

$$
y^{\mu \lambda} \nabla_{\mu} \nabla_{\lambda} f+y^{\mu \lambda}\left(\nabla_{\mu} \varphi_{\lambda}{ }^{\varepsilon}\right) \varphi_{\varepsilon}{ }^{v} \nabla_{v} f=0,
$$

which and Hopf's theorem ${ }^{5}$ yield the Lemma.

This lemma and the definition of almost-analytic function lead us to

THEOREM 1. In a compact almost-complex space an almost-analytic function is constant.

The following lemma is also known ${ }^{6)}$

LEMMA 2. If $T_{(\mu)}^{(\mathcal{l})} \equiv T_{\mu_{q} \ldots \mu_{1}}^{\lambda_{p} \ldots \lambda_{1}}$ of type $(p, q)(\neq(1,0))$ is almost-analytic, then so is $\stackrel{*}{T}_{(\mu)}^{(\lambda)}$.

Now let $T_{(\mu)}^{(\lambda)}$ be pure, $\rho$ an $\sigma$ be scalar functions. If we operate $\Phi_{\nu}$ to $\rho T_{(\mu)}^{(\lambda)}+\sigma \stackrel{*}{T}_{(\mu)}^{(\lambda)}$, we have easily

$$
\begin{aligned}
\Phi_{\nu}\left(\rho T_{(\mu)}^{(\lambda)}\right. & \left.+\sigma \stackrel{*}{T}_{(\mu)}^{(\lambda)}\right)=\rho \Phi_{\nu} T_{(\Lambda)}^{(\lambda)}+\sigma \Phi_{\nu} \stackrel{*}{T}_{(\mu)}^{(\lambda)} \\
& +\left(\varphi_{\nu}^{\varepsilon} \partial_{\varepsilon} \rho+\partial_{\nu} \sigma\right) T_{(\mu)}^{(\lambda)}+\left(\varphi_{\nu}^{\varepsilon} \partial_{\varepsilon} \sigma-\partial_{\nu} \rho\right) \stackrel{*}{T}_{(u)}^{(\lambda)} .
\end{aligned}
$$

5) Yano, K. and S. Bochner, [7].

6) Tachibana, S., [6], Kotō, S., [3]. 
Taking account of the fact that $T_{(\mu)}^{(\lambda)}$ and $\stackrel{T}{(\mu)}^{(\lambda)}$ are linearly independent we get by virtue of Lemma 1 and Lemma 2 the following

THEOREM 2. Let $T_{(\mu)}^{(\lambda)}$ of type $(p, q)(\neq(1,0))$ be almost-analytic in a compact almost-complex space. Then a necessary and sufficient condition for $\rho T_{(\mu)}^{(\lambda)}+\sigma \stackrel{*}{T}_{(i)}^{(\lambda)}$ to be almost-analytic is that $\rho$ and $\sigma$ are constant.

It is known that for pure tensors $V_{(\mu)}{ }^{(\lambda)}$ and $U_{(\lambda)}{ }^{(\mu)}$ we have ${ }^{7)}$

$$
\begin{aligned}
V_{(\mu)}^{(\lambda)} \Phi_{\nu} U_{(\lambda)}^{(\mu)} & +U_{(\lambda)}^{(\mu)} \Phi_{\nu} V_{(\mu)}^{(\lambda)} \\
& =\phi_{\nu}{ }^{\varepsilon} \partial_{\varepsilon}\left(V_{(\mu)}{ }^{(\lambda)} U_{(\lambda)}^{(\mu)}\right)-\partial_{\nu}\left(V_{(\mu)}^{*}{ }^{(\lambda)} U_{(\lambda)}^{(\mu)}\right),
\end{aligned}
$$

so we have

THEOREM 3. In an almost-complex space, the scalar function defined by the inner product of two almost-analytic tensors is almost-analytic.

2. Almost-analytic submanifolds. We consider an almost-complex space $X_{n}$ and an $m$-dimensional submanifold $X_{m}$ expressed locally by the equation

$$
x^{\lambda}=x^{\lambda}\left(\xi^{a}\right), \quad \operatorname{rank}\left(\partial_{a} x^{\lambda}\right)=m,
$$

where Greek indices take the values 1 to $n$, Latin indices the values 1 to $m(<n)$ and $\partial_{a}=\partial / \partial \xi^{a}$.

Now we assume that $X_{m}$ is an almost-complex space too and denote its structure tensor by $\varphi_{b}{ }^{a}$. Then we can define locally almost-analytic functions on $X_{m}$ by $\varphi_{b}{ }^{a}$ as analogous as in $\S 1$. An almost-complex space $X_{m}$ will be called an almost-analytic submanifold, if $f\left(x^{\lambda}\left(\xi^{a}\right)\right)$ is always almost-analytic of $\xi^{a}$ for any almost-analytic function $f$ of $x^{\lambda}$. If $X_{n}$ and $X_{m}$ are complex manifolds, then we call an almost-analytic submanifold an analytic submanifold.

On the other hand a submanifold $X_{m}$ is called an invariant subspace, ${ }^{8)}$ if its $m$-dimensional tangent plane at any point remains invariant under the transformation $v^{\lambda} \rightarrow v^{*}=\varphi_{\varepsilon}^{\lambda} v^{s}$.

Suppose that $X_{m}$ be an invariant subspace. Then as $m$ vectors $B_{a}{ }^{\lambda}=\partial x^{\lambda} / \partial \xi^{a}$ $(a=1,2, \ldots, m)$ span the tangent plane at each point of $X_{m}$, there exists a tensor field $\varphi_{b}{ }^{a}$ such that

$$
\boldsymbol{\varphi}_{\varepsilon}^{\lambda} B_{a}{ }^{\varepsilon}=\varphi_{a}{ }^{c} B_{c}^{\lambda} .
$$

From this equation we can see $p_{c}{ }^{a} \phi_{b}{ }^{c}=-\delta_{b}{ }^{a}$, so an invariant subspace becomes an almost-complex space by virtue of the induced tensor $\varphi_{b}{ }^{a}$. Let $f$ and $g$ be a locally almost-analytic function and its associated function, respectively. Putting $F\left(\xi^{a}\right)=f\left(x^{\lambda}\left(\xi^{a}\right)\right)$ and $G\left(\xi^{a}\right)=g\left(x^{\lambda}\left(\xi^{a}\right)\right)$ we have

7) Tachibana, S., [6].

8) Schouten, J.A. and K. Yano, [5]. 


$$
\begin{aligned}
\varphi_{c}{ }^{b} \partial_{b} F & =\varphi_{c}^{b} B_{b}^{s} \partial^{\varepsilon} f=\varphi_{\lambda}^{\varepsilon} B_{c}^{\lambda} \partial^{\varepsilon} f \\
& =B_{c}^{\lambda} \partial_{\lambda} g=\partial_{c} G,
\end{aligned}
$$

which means that $F$ is an almost-analytic function of $\xi^{a}$. Thus we get the following

THEOREM 4. An invariant subspace in an almost-complex space is an almost-analytic submanifold by virtue of the induced almost-complex structure.

For a locally almost-analytic $f$ and its associated fnuction $g$ we consider the equations

$$
f\left(x^{\imath}\right)=\text { const.; } \quad g\left(x^{\imath}\right)=\text { const.. }
$$

As $f$ and $g$ are functionally independent, they define locally an $(n-2)$-dimensional subspace. Since $v^{\lambda} \partial_{\lambda} f=v^{\lambda} \partial_{\lambda} g=0$ imply ${ }_{v}^{*} \partial_{\lambda} f=\stackrel{*}{v}^{\lambda} \partial_{\lambda} g=0$, the subspace is invariant. Thus we have

THEOREM 5. In an almost-complex space if an $(n-2)$-dimensional submanifold is representable locally by the equations $f\left(x^{\lambda}\right)=$ const., $g\left(x^{\lambda}\right)=$ const., where $f$ and $g$ be a locally almost-analytic function and its associated function respectively, then it is an almost-analytic submanifold by virtue of the induced almosi-complex structure.

Let $v^{\lambda}$ be a (contravariant) vector field, then $v^{\lambda}$ and ${ }^{*}{ }^{\lambda}$ span an invariant plane at each point such that $v^{\lambda} \neq 0$. We call this field of 2 -planes the distribution associated to $v^{\lambda}$. Now we suppose $v^{\lambda}$ is almost-analytic, then we have $\underset{v}{f}, \phi_{\mu}^{\lambda}=0$ and

$$
\left[v, v_{v}^{*}\right]^{\lambda}=\underset{v}{\mathfrak{f}} \stackrel{v}{v}_{\lambda}^{*}=\underset{v}{\mathfrak{f}}\left(\phi_{\varepsilon}^{\lambda} v^{\varepsilon}\right)=\phi_{\varepsilon}^{\lambda} \underset{v}{\mathfrak{L}} v^{s}=0 .
$$

This equation means that the distribution is involutive.9) As its plane elements are invariant we get

THEOREM 6. The 2-dimensional distribution associated to an almostanalytic vector field $v^{\lambda}$ is involutive and its integral manifolds are almostanalytic by virtue of the induced structure.

3. Tensors of mixed kind on subspaces. We consider an $n$-dimensional differentiable manifold $X_{n}$ and $m$-dimensional submanifold $X_{m}$ expressed locally by $x^{\lambda}=x^{\lambda}\left(\xi^{a}\right)$. We assume the existence of $\varphi_{\lambda}{ }^{k}$ and $\varphi_{b}{ }^{a}$ which are tensors of type $(1,1)$ in $X_{n}$ and $X_{m}$ respectively. By a tensor of mixed kind $T_{(b)(\mu)}{ }^{(a)(\lambda)}$ $\equiv T_{b_{q} \ldots b_{1} \mu_{s} \ldots \mu_{1}} a_{j \ldots} \ldots a_{1} \lambda_{\ldots} \ldots \lambda_{1}$ we mean a quantity defined on $X_{m}$ that it is a tensor field of type $(r, s)$ in $X_{n}$ for fixed indices $(a)$ and $(b)$ and is a tensor field of type $(p, q)$ in $X_{m}$ for fixed indices $(\lambda)$ and $(\mu)$. For simplicity we call it a tensor of type $(r, s ; p, q)$.

9) Chevally, C., [1]. 
If $T_{\left({ }^{\prime}\right)(\mu)}{ }^{(a)(\lambda)}$ commutes with $\varphi_{\mu}{ }^{\lambda}$ and ${\varphi_{b}}^{a}$ in its two indices, then we say that it is pure in the indices. More precisely speaking it is pure in $b_{i}, b_{j}$, for simplicity we denote it $\mathfrak{p}\left(b_{i}, b_{j}\right)$, if

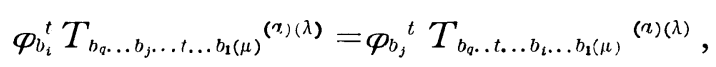

and is $\mathfrak{p}\left(b_{j}, \mu_{i}\right)$ if

$$
\phi_{h_{j}}{ }^{t} T_{b_{q} \ldots t \ldots b_{1}(\mu)}{ }^{(a)(1)}={\phi_{\mu_{t}}}^{\varepsilon} T_{(1) \mu_{s} \ldots \varepsilon \ldots \mu_{1}}^{(a)(\lambda)},
$$

and is $\mathfrak{p}\left(a_{j}, \mu_{i}\right)$ if

$$
\varphi_{t}^{a_{j}} T_{(b)(\mu)}^{a_{p} \ldots{ }^{+} \ldots a_{1}(\lambda)}={\varphi_{\mu_{\imath}}}^{\varepsilon} T_{(b) \mu_{\imath} \ldots \varepsilon \ldots \mu_{1}}^{(a)(\lambda)},
$$

and so on.

If a tensor is pure in all pairs of its indices, then it is called a pure tensor. The vector $v^{b}$ or $u_{c}$ on $X_{m}$ or $V^{\lambda}$ or $U_{\mu}$ restricted to $X_{m}$ is considered to be pure, by convention.

The tensor fields $\delta_{\mu}^{\lambda},{\varphi_{\mu}}^{\lambda}, \delta_{b}{ }^{a}$ and $\varphi_{b}{ }^{a}$ are pure. If $T_{(b)(\mu)}^{(\alpha)(\lambda)}$ anti-commutes with $\varphi_{\lambda}{ }^{\mu}$ and ${\varphi_{b}}^{a}$ in its two indices, then we say it is hybrid in the indices. For instance if

$$
\varphi_{b}{ }^{t} T_{b_{q} \ldots+\ldots b_{t}(\mu)}{ }^{(a)(\lambda)}=-\phi_{\varepsilon}^{\lambda^{\lambda}} T_{(b)(\mu)}{ }^{(a) \lambda_{r} \ldots \varepsilon_{1} \ldots \lambda_{1}}
$$

hold good, then it is hybrid in $b_{j}, \lambda_{l}$. We denote this fact by $\mathfrak{h}\left(b_{j}, \lambda_{l}\right)$.

The following facts are easily seen. ${ }^{10)}$

If $T_{b \mu}{ }^{a \lambda}$ is $\mathfrak{p}(a, b)$ (or $\left.\mathfrak{h}(a, b)\right)$ and also $\mathfrak{p}(\lambda, b)$ (or $\left.\mathfrak{h}(\lambda, b)\right)$, then it is $\mathfrak{p}(a, \lambda)$ etc..

If $T_{b \mid l}{ }^{a \lambda}$ is $\mathfrak{p}(a, b)$ and $\mathfrak{h}(\lambda, b)$, then it is $\mathfrak{h}(a, \lambda)$, etc..

If $T_{b j i}{ }^{a \lambda}$ and $U_{a \lambda}{ }^{c k}$ are $\mathfrak{p}(a, \lambda)$ (or $\mathfrak{h}^{\prime}(a, \lambda)$ ), then $T_{b{ }^{a}}{ }^{a} U_{a \nu}{ }^{c \kappa}$ is $\mathfrak{p}(\lambda, u)$, etc..

If $T_{b, \imath}{ }^{a \lambda}$ is $\mathfrak{p}(a, \lambda)$ and $U_{a \lambda}{ }^{c \kappa}$ is $\mathfrak{h}(a, \lambda)$, then $T_{b \mu}{ }^{a \lambda} U_{a}{ }^{c \kappa}$ is $\mathfrak{h}(\lambda, \nu)$, etc..

If $T_{b \mu}^{a \lambda}$ is pure in some indices, then so is ${\stackrel{*}{T_{b \mu}}}^{a \lambda}=T_{\mu}^{a \lambda} \phi_{b}{ }^{t}$, and so on.

4. $\Phi$-operator. Let $X_{n}$ be a differentiable manifold admitting a tensor field $\varphi_{\mu}^{\lambda}$ and $X_{m}$ be a submanifold represented locally by $x^{\lambda}=x^{\lambda}\left(\xi^{a}\right)$. An $m$-plane in the tangent space at a point of $X_{n}$ is called invariant if it contains its image under the transformation $v^{\lambda} \rightarrow v^{*} \lambda=\varphi_{\varepsilon}^{\lambda} v^{\varepsilon}$. If the tangent $m$-plane at any point of $X_{m}$ is invariant, we call $X_{m}$ an invariant subspace.

Let $X_{m}$ be an invariant subspace. As $m$ vectors $B_{a}{ }^{\lambda}=\partial x^{\lambda} / \partial \xi^{a}(a=1,2, \ldots \ldots$, $m)$ in $X_{n}$ span the tangent $m$-plane of $X_{m}$, their images are linear combinations of themselves. Hence there exists a tensor field $\varphi_{b}{ }^{a}$ on $X_{m}$ such that

$$
\varphi_{s}^{\lambda} B_{a}^{\tau}=\varphi_{a}{ }^{c} B_{c}{ }^{\lambda} .
$$

Conversely if there exists a tensor field ${\varphi_{b}}^{a}$ satisfying (4.1), then $X_{m}$ is invariant. We shall call $\varphi_{b}{ }^{a}$ satisfying (4.1) the induced $\varphi_{b}{ }^{a}$ or the induced structure.

10) cf. Tachibana, S., [6]. Kotō, S., [3]. 
The equation (4.1) means that $B_{a}{ }^{\lambda}$ of an invariant subspace is pure with respect to ${\varphi_{\mu}}^{\lambda}$ and the induced $\varphi_{b}{ }^{a}$.

In the following we introduce an operator $\Phi$ on an invariant $X_{m}$ which is associated to $\varphi_{\mu}{ }^{\lambda}$ and the induced $\varphi_{b}{ }^{a}$. This operator will yield from a pure tensor of type $(r, s ; p, q)$ a new tensor of type $(r, s ; p, q+1)$.

Now we define an operator $\Phi$ acting on a pure tensor $T_{(b)(\mu)}{ }^{(a)(\lambda)}$ by the following equation,

$$
\begin{aligned}
& \Phi_{c} T_{(b)(\mu)}^{()^{(\lambda)}(\lambda)}=\varphi_{c}{ }^{l} \partial_{l} T_{(b)(\mu)}^{(a)(\lambda)}-\partial_{c}{ }^{*}{ }_{(b)(\mu)}{ }^{(a)(\lambda)} \\
& +\sum_{j=1}^{q}\left(\partial_{b_{j}} \boldsymbol{\varphi}_{c}{ }^{l}\right) T_{b_{q} \ldots l \ldots b_{1}(\mu)}{ }^{(a)(\lambda)}+\sum_{i=1}^{p}\left(\partial_{c}{\boldsymbol{\varphi}_{l}}^{a_{l}}-\partial_{l}{\boldsymbol{\varphi}_{c}}^{a)}\right) T_{(b)(\mu)}{ }^{a_{p} \ldots l \ldots a_{1}(\lambda)} \\
& +\sum_{j=1}^{s}\left(\partial_{\mu_{j}}{\varphi_{\varepsilon}}^{\sigma}\right) B_{c}{ }^{\tau} T_{(b) \mu_{s} \ldots \sigma \ldots \mu_{1}}^{(a)(\lambda)} \\
& +\sum_{i=1}^{r}\left(\partial_{\varepsilon}{\mathcal{P}_{\sigma}}^{\lambda_{t}}-\partial_{\sigma}{\varphi_{\varepsilon}}^{\lambda_{i}}\right) B_{c}^{\varepsilon} T_{(b)(\mu)}^{(a) \lambda_{r} \ldots \sigma \ldots \lambda_{1}},
\end{aligned}
$$

where we put

$$
\stackrel{*}{T}_{(a)(\mu)}^{(b)(\lambda)}=\phi_{b_{j}}{ }^{l} T_{b_{q} \ldots \ldots b_{1}(\mu)}{ }^{(a)(\lambda)}={\varphi_{\varepsilon}}^{\lambda,} T_{(b)(\mu)}{ }^{(a) \lambda_{r} \ldots \varepsilon_{1} \ldots \lambda_{1}},
$$

etc., on taking account of the purity.

We must prove the tensor property of the operator. To do it we introduce any affine connections $\Gamma_{\mu \nu}{ }^{\lambda}$ and $\Gamma_{b c}{ }^{a}$ in $X_{n}$ and $X_{m}$ respectively and denote by $\nabla_{v}$ and $\nabla_{c}$ the corresponding operators of covariant derivation. Of course $\nabla_{c}$ operates on, for instance, as follows,

$$
\begin{aligned}
& \nabla_{c} T_{b}{ }^{a}=\partial_{c} T_{b}{ }^{a}+T_{b}{ }^{l} \Gamma_{c l}{ }^{a}-T_{l}{ }^{a} \Gamma_{c b}{ }^{l}, \\
& \nabla_{c} T_{b \mu}{ }^{\lambda}=\partial_{c} T_{b ; l}{ }^{\lambda}-T_{l \mu}{ }^{\lambda} \Gamma_{c b}{ }^{l}+B_{c}{ }^{\varepsilon}\left(T_{b j}{ }^{\lambda} \Gamma_{\varepsilon \mu}{ }^{\sigma}-T_{b \mu}{ }^{\sigma} \Gamma_{\varepsilon \sigma}{ }^{\lambda}\right) .
\end{aligned}
$$

Denoting the torsion tensors by

$$
S_{\nu \mu}^{\lambda}=(1 / 2)\left(\Gamma_{\nu \mu}^{\lambda}-\Gamma_{\mu \nu}^{\lambda}\right), \quad S_{c b}^{a}=(1 / 2)\left(\Gamma_{c b}^{a}-\Gamma_{b c}^{a}\right)
$$

we have

$$
\begin{aligned}
& \partial_{b,} \boldsymbol{\varphi}_{c}{ }^{l}=\nabla_{b_{j}} \boldsymbol{\varphi}_{c}{ }^{l}-\boldsymbol{\varphi}_{c}{ }^{t} \Gamma_{b_{j} t}{ }^{l}+\boldsymbol{\varphi}_{l}{ }^{l} \Gamma_{b_{j},}{ }^{t}, \\
& \partial_{c} \boldsymbol{\varphi}_{l}^{a_{t}}-\partial_{l} \boldsymbol{\varphi}_{c}{ }^{a_{i}}=\nabla_{c} \boldsymbol{\varphi}_{l}{ }^{a_{i}}-\nabla_{l} \boldsymbol{\varphi}_{c}{ }^{a_{i}}-\boldsymbol{\varphi}_{l}{ }^{t} \Gamma_{c t}{ }^{a_{t}} \\
& +\boldsymbol{\varphi}_{c}{ }^{t} \Gamma_{l t}{ }^{a_{i}}+2 \boldsymbol{\varphi}_{t}{ }^{a_{i}} S_{c l}{ }^{t}, \\
& \partial_{\mu_{j}} \phi_{\varepsilon}{ }^{\sigma}=\nabla_{\mu_{j}} \phi_{\varepsilon}{ }^{\sigma}+\varphi_{\tau}^{\sigma} \Gamma_{\mu_{j} \varepsilon}^{\tau}-\varphi_{\varepsilon}{ }^{\tau} \Gamma_{\mu_{j} \tau}^{\sigma}, \\
& \partial_{\varepsilon} \varphi_{\sigma}^{\lambda_{i}}-\partial_{\sigma} \varphi_{\varepsilon}^{\lambda_{i}}=\nabla_{\varepsilon} \varphi_{\sigma}^{\lambda_{i}}-\nabla_{\sigma}{\varphi_{\varepsilon}}^{\lambda_{i}}-\varphi_{\sigma}^{\tau} \Gamma_{\varepsilon \tau}{ }^{\lambda_{i}} \\
& +\varphi_{\varepsilon}{ }^{\tau} \Gamma_{\sigma \tau}^{\lambda^{\lambda_{i}}}+2{\varphi_{\tau}}^{\lambda^{\lambda_{i}}} S_{\varepsilon \sigma}{ }^{\tau} \text {. }
\end{aligned}
$$

Substituting these relations into (4.2) and taking account of the purity of $B_{a}{ }^{\lambda}$ 
and $T_{(b)(\mu)}{ }^{(a)(\lambda)}$ we have

$$
\begin{aligned}
& \Phi_{c} T_{(b)(\mu)}^{(a)(\lambda)}=\phi_{c}{ }^{l} \Delta_{l} T_{(b)(\mu)}^{(a)(\lambda)}-\nabla_{c} \stackrel{*}{T}_{(b)(\mu)}^{(\lambda)(1)} \\
& +\sum_{j=1}^{q}\left[\nabla_{b}, \boldsymbol{\varphi}_{c}{ }^{l}+2\left(S_{b_{j},{ }^{t}} \boldsymbol{\varphi}_{t}{ }^{l}-S_{b_{j}}{ }^{l} \boldsymbol{\varphi}_{c}{ }^{t}\right)\right] T_{b_{q} \ldots l \ldots b_{1}(\mu)}{ }^{(a)(\lambda)} \\
& +\sum_{i=1}^{p}\left[\nabla_{c}{\varphi_{l}}^{a_{i}}-\nabla_{l}{\varphi_{c}}^{a_{i}}+2\left(S_{c l}{ }^{t} \phi_{t}^{a_{t}}-S_{t l}{ }^{a_{i}} \phi_{c}{ }^{t}\right)\right] T_{(b)(\mu)}{ }^{a_{p} \ldots l \ldots a_{1}(\lambda)}
\end{aligned}
$$

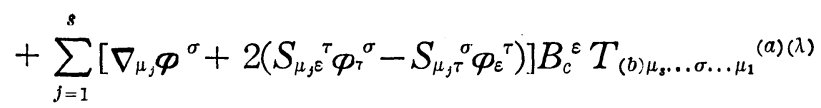

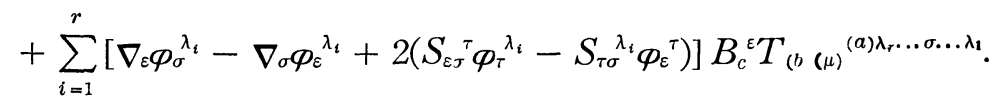

This shows that $\Phi_{c} T_{(b)(\mu)}^{(x)(\lambda)}$ is a tensor and hence the $\Phi$-operator has the tensor property.

5. Some formulas. From (4. 2) we have

$$
\begin{aligned}
\Phi_{c} T_{(b)}{ }^{(a)} & =\varphi_{c}{ }^{l} \partial_{l} T_{(b)}{ }^{(a)}-\partial_{c} T_{(b)}{ }^{(a)} \\
& +\sum_{j=1}^{q}\left(\partial_{b_{j}} \varphi_{c}{ }^{l}\right) T_{b_{q} \ldots l \ldots b_{1}}{ }^{(a)}+\sum_{i=1}^{p}\left(\partial_{c}{\varphi_{l}}^{a_{i}}-\partial_{l}{\varphi_{c}}^{a_{i}}\right) T_{(b)}{ }^{a_{p} \ldots l \ldots a_{1}}
\end{aligned}
$$

for a pure tensor $T_{(b)}^{(a)}$ in $X_{m}$, which corresponds to the $\Phi_{v}$-operator in $X_{n}$ in $\S 1$. If we consider a pure tensor $T_{(\mu)}^{(\lambda)}$ of $X_{n}$ on $X_{m}$ and operate $\Phi_{c}$ to it, then we have

$$
\Phi_{c} T_{(\mu)}^{(\lambda)}=B_{c}{ }^{\varepsilon} \Phi_{\varepsilon} T_{(\mu)}^{(\lambda)} .
$$

We can also prove the following formula

$$
\Phi_{c}\left(V_{b}^{\varepsilon} U_{\varepsilon}^{\lambda}\right)=\left(\Phi_{c} V_{b}^{\varepsilon}\right) U_{\varepsilon}^{\lambda}+V_{b}^{\varepsilon} \Phi_{c} U_{\varepsilon}^{\lambda}
$$

whose analogous formula is valid for pure tensors of the most general type too.

As $B_{a}{ }^{\lambda}$ is pure we can operate $\Phi_{c}$ to it and then we have

$$
\begin{aligned}
& \Phi_{c} B_{a}{ }^{\lambda}=\varphi_{c}{ }^{l} \partial_{l} B_{a}{ }^{\lambda}-\partial_{c}\left(\varphi_{a}{ }^{l} B_{l}{ }^{\lambda}\right)+\left(\partial_{a} \varphi_{c}{ }^{l}\right) B_{l}{ }^{\lambda} \\
& +\left(\partial_{\varepsilon} \varphi_{\sigma}^{\lambda}-\partial_{\sigma} \phi_{\varepsilon}^{\lambda}\right) B_{c}{ }^{\varepsilon} B_{a}{ }^{\sigma} \\
& =\partial_{a}\left(\phi_{c}{ }^{l} B_{l}^{\lambda}\right)-\partial_{c}\left(\phi_{a}{ }^{l} B_{l}^{\lambda}\right)+\left(\partial_{\varepsilon}{\varphi_{\sigma}}^{\lambda}-\partial_{\sigma}{\varphi_{\varepsilon}}^{\lambda}\right) B_{c}{ }^{\varepsilon} B_{a}{ }^{\sigma} .
\end{aligned}
$$

If we differentiate $\varphi_{c}{ }^{l} B_{l}{ }^{\lambda}={\varphi_{\varepsilon}}^{\lambda} B_{c}{ }^{\varepsilon}$ with respect to $\xi^{a}$, then we have

$$
\partial_{a}\left(\varphi_{c}{ }^{l} B_{l}^{\lambda}\right)-\left(\partial_{\sigma}{\varphi_{\varepsilon}}^{\lambda}\right) B_{c}{ }^{\varepsilon} B_{a}{ }^{\sigma}=\varphi_{\varepsilon}{ }^{\lambda} \partial_{a} B_{c}^{\varepsilon} \text {. }
$$

Interchanging $a$ and $c$ in (5.1) and substracting the equation thus obtained from (5.1) we get 


$$
\Phi_{c} B_{a}^{\lambda}=\Phi_{c}\left(\frac{\partial x^{\lambda}}{\partial \xi^{a}}\right)=0 .
$$

This corresponds to $\Phi_{\nu} \delta_{\mu}{ }^{\lambda}=0$ in $\S 1$.

Next as the both hand members of $\varphi_{c}{ }^{a} B_{a}{ }^{\lambda}={\varphi_{i}}^{\lambda} B_{c}{ }^{\varepsilon}$ are pure, we have $B_{a}{ }^{\lambda} \Phi_{b} \varphi_{c}{ }^{a}=B_{c}{ }^{\varepsilon} B_{b}{ }^{\sigma} \Phi_{\sigma}{\varphi_{\varepsilon}}^{\lambda}$. From which we get

$$
N_{b c}{ }^{a} B_{a}{ }^{\lambda}=N_{\sigma \varepsilon}{ }^{\lambda} B_{b}{ }^{\sigma} B_{c}{ }^{\varepsilon},
$$

where $N_{b c}{ }^{a}$ and $N_{\sigma \varepsilon}{ }^{\lambda}$ are the Nijenhuis tensors of ${\varphi_{b}}^{a}$ and $\varphi_{\mu}{ }^{\lambda}$ respectively. Thus we have

THEOREM 7. ${ }^{11)}$ In a differentiable manifold admitting a tensor field $\varphi_{\mu}{ }^{\lambda}$, let $\phi_{b}{ }^{a}$ be the induced structure on an invariant subspace. Then if the Nijenhuis tensor of ${\varphi_{\mu}{ }^{\lambda}}$ vanishes, the Nijenhuis tensor of ${\varphi_{b}}^{a}$ vanishes too.

REMARK. In the definition of $\Phi$-operator we need not assume that $m<n$. In the case $n=m, x^{\lambda}=x^{\lambda}\left(\xi^{a}\right)$ is considered as a transformation of local coordinates.

6. Kählerian spaces. An almost-complex space is called a complex manifold if the Nijenhuis tensor vanishes identically. About such a space we get from Theorem 4 and Theorem 7 the following

THEOREM 8. An invariant subspace in a complex manifold is an analytic submanifold by virtue of the induced structure.

An almost-complex space is called almost-Hermitian if it is a Riemannian space and that the Riemannian metric $g_{\mu \lambda}$ is hybrid with respect to the almostcomplex structure $\varphi_{\mu}{ }^{\lambda}$. In such a space since the tensor field $\phi_{\mu \lambda}=\varphi_{\mu}{ }^{\varepsilon} g_{\varepsilon \lambda}$ is antisymmetric we have the so-called fundamental form $\varphi=\phi_{\mu \lambda} d x^{u} \wedge d x^{\lambda}$. A Kählerian space is an almost-Hermitian space in which $\nabla_{\nu} \varphi_{\mu}{ }^{\lambda}=0$ is valid, where $\nabla_{v}$ denotes the operator of the Riemannian covariant derivation. It is characterized as the almost-Hermitian space such that it is a complex manifold and that the fundamental form is closed. ${ }^{12)}$

In the following we shall only consider a Kählerian space $X_{n}$ and its invariant subspace $X_{m}$. We denote by $g_{c b}$ the induced Riemannian metric defined by $g_{c b}=g_{\mu \lambda} B_{b}{ }^{\mu} B_{c}{ }^{\lambda}$. As $g_{\mu \lambda}$ is hybrid and $B_{b}{ }^{\mu}$ is pure, we see that $g_{c b}$ is hybrid, on account of the arguments in $\S 3$. Hence $X_{m}$ becomes almost-Hermitian. By the relation $\varphi_{c b}=\varphi_{c}{ }^{r} g_{r b}=\varphi_{\mu \lambda} B_{c}{ }^{\mu} B_{b}{ }^{\lambda}$ we know that the fundamental form of $X_{m}$ is closed. Thus if we take account of Theorem 7 we get the well known

TheOREM 9. ${ }^{13)}$ An invariant subspace in a Kählerian space is itself Kählerian by virtue of the induced structure.

11) cf. Schouten, J.A. and K. Yano, [5].

12) Schouten, J.A. and K. Yano, [5], Kotō, S., [2].

13) Schouten, J.A. and K. Yano, [5]. 
As we have $\nabla_{b} \varphi_{c}{ }^{a}=0$ in an invariant $X_{m}$ in a Kählerian $X_{n}$, the equation (4. 3) turns into the following simple form,

$$
\Phi_{c} T_{(b)(\mu)}^{(a)(1)}={\phi_{c}}^{l} \nabla_{l} T_{(b)(\mu)}^{(a)(\lambda)}-\nabla_{c} \stackrel{*}{T}_{(b)(\mu)}^{(a)(1)} .
$$

Hence $\Phi_{c} T_{(b)(i)}^{(a)(\lambda)}=0$ for a pure tensor is equivalent to that $\nabla_{c} T_{(b)(\mu)}^{(a)(\lambda)}$ is also pure.

An infinitesimal conformal transformation $v^{\lambda}$ in $X_{n}$ is a vector field such that $\underset{v}{\mathfrak{f}} g_{\mu \lambda}=2 \rho g_{\mu \lambda}$, where $\rho$ is a scalar function. An infinitesimal projective transformation is a vector field $v^{\lambda}$ such that $\underset{v}{\mathfrak{L}}\left\{\begin{array}{c}\lambda \\ \mu \nu\end{array}\right\}=\rho_{\mu} \delta_{\nu}{ }^{\lambda}+\rho_{\nu} \delta_{\mu}{ }^{\lambda}$, where $\left\{\begin{array}{c}\lambda \\ \mu \nu\end{array}\right\}$ means the Christoffel symbols and $\rho_{\mu}$ is necessarily gradient. Recently Y.Tashiro ${ }^{14)}$ showed that $\rho_{\nu}=\partial_{\nu} \rho$ is (covariant) almost-analytic for these transformations. This means by our terminology that $\rho$ is an almost analytic function. So there exists a family of its associated functions $\{\sigma\}$ and if two functions of the family have a common domain of definition, then their difference is constant. Now we assume that the transformation in consideration is not homothetic i.e. that $\rho$ is not constant. We consider a family of local submanifolds $\sigma=$ const., so they define globally a family of $(n-1)$-dimensional submanifolds. The intersection of $\rho=$ const. and a submanifold of the family is invariant by accordance of the argument in $\S 2$. Thus we have

THEOREM 10. If a Kählerian space admits a non-homothetic infinitesimal conformal (or projective) transformation, then there exist $\infty^{2}(n-2)$-dimensional invariant subspaces, each of which is an analytic submanifold and Kählerian by virtue of the induced structure.

\section{BIBLIOGRAPHY}

[1] Chevally, C., Theory of Lie groups I, Princeton Univ. Press., 1946.

[2] Kơro, S., Some theorems on almost Kählerian spaces, Jour. Math. Soc. Japan, 12(1960), 422-433.

[3] Kото̄, S., On almost analytic tensors in almost complex spaces, to appear in Tensor.

[4] NiJENHUIS, A., $X_{n-1}$-forming sets of eigenvectors, Indag. Math., 13(1951), 200-212.

[5] Schouten, J.A. AND K. YANO, On invariant subspaces in the almost complex $X_{2 n}$, Indag. Math., 17(1955), 261-269.

[6] TACHIBANA, S., Analytic tensor and its generalization, Tôhoku Math. Jour., 12 (1960), 208-221.

[7] YANO, K. AND S. BochNER, Curvature and Betti numbers, Annals of Math. Studies, $32(1953)$.

OCHANOMIZU UNIVERSITY, TOKYO AND NIIGATA UNIVERSITY.

14) Personal communication. 